\title{
ELECTRON PARAMAGNETIC RESONANCE STUDY OF Se-DOPED AlSb: EVIDENCE FOR NEGATIVE-U OF THE DX CENTER
}

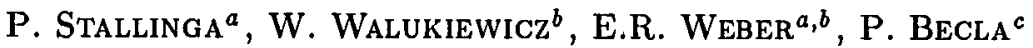 \\ AND J. LAGOWSKI ${ }^{d}$ \\ ${ }^{a}$ Department of Materials Science and Mineral Engineering \\ University of California at Berkeley, Berkeley, CA 94720, USA \\ ${ }^{b}$ Materials Science Division, Lawrence Berkeley Laboratory, Berkeley, CA 94720, USA \\ ${ }^{c}$ Department of Materials Science, Massachusetts Institute of Technology \\ Cambridge, MA 02139, USA \\ ${ }^{d}$ Center for Microelectronics Research, University of South Florida \\ Tampa, FL 33620, USA
}

A metastable electron paramagnetic resonance signal is observed in Se doped AlSb. After illumination a strong, persistent electron paramagnetic resonance signal with an isotropic $g$-factor of 1.949 is observed. The absence of any electron paramagnetic resonance when cooled in the dark is direct evidence for the negative- $U$ model. The electron paramagnetic resonance arises from the effective-mass state of the defect, which is not filled at thermal equilibrium. An analysis of the lineshape reveals that the linewidth is determined by hyperfine interactions. The extend of the wave function is found to be comparable to the prediction for the effective-mass state.

PACS numbers: 71.55.Eq, 76.30.Da.

\section{Introduction}

After the discovery of the DX center by Lang et al. [1], it has gained interest among scientists in industry and academia. This is mostly due to the fact that it is omnipresent in compound semiconductors and their alloys [2] and, in most cases, it dominates the electrical properties of the material. What is more, the structural metastability of the defect causes a metastability of the properties of the semiconductor. This structural metastability consists of the two stable positions of the donor atom, it can either reside on the substitutional lattice position $\left(T_{\mathrm{d}}\right)$ where it acts as a hydrogenic donor atom or the atom and/or surrounding host lattice can relax, and thus form a new ( $C_{3 v}$ symmetry) ground state.

Theoretical calculations performed for GaAs [3] have shown that group-IV and group-VI donors undergo different types of structural relaxation upon electron 
capture. If the donor is a group-IV atom on a group-III sublattice, the donor itself is displaced from the substitutional site to an interstitial site, whereas for a group-VI on the group-V sublattice, one of the surrounding Ga-atoms is displaced along the 〈111) direction. In both cases a vacant Ga site is formed. A conclusion of this model is that the localized DX state exhibits an effective negative electron correlation energy (negative-U) resulting in a diamagnetic ground state with paired-off spins.

In the negative- $U$ model paramagnetic, neutral centers $d^{0}$ never exist, since they are spontaneously transformed into the non paramagnetic configurations $d^{+}$ and $D X^{-}$in the exothermic reaction [3]

$$
2 d^{0} \rightarrow d^{+}+D X^{-} \text {. }
$$

In this case the negatively charged $D X^{-}$relaxes to a $C_{3 v}$ symmetry.

In a positive-U model, however, the $d^{0}$ state of the hydrogenic donor can always be realized by a suitable adjustment of the Fermi level. Thus, the absence of an EPR signal in an $n$-type semiconductor after freezing out of the carriers is direct evidence for a negative-U situation.

For most of the binary III-V semiconductors, the DX level is resonant with the conduction band. This makes it very hard to measure. The solution is to apply hydrostatic pressure [4], or, equivalently, use ternary compounds. Both methods shift the DX level into the bandgap, where they can serve as efficient electron traps. For GaAs this occurs at pressures above $20 \mathrm{kbar}$ or for more than 22 at\% $\mathrm{Al}$ content in $\mathrm{AlGaAs}$. Both methods are cumbersome and results of various experiments were often inconclusive or contradictory.

Apart from the deep DX level the donor forms the usual shallow, effective-mass level [4] connected to the minimum of the conduction band. The depth of this level depends on the type of material and the valley to which it is connected, but is usually in the order of $10 \mathrm{meV}$. Because they are so shallow, they will be emptied into the conduction band for most temperatures. This warrants the use of AlSb for studying DX; it is an easy-to-grow-bulk binary III-V semiconductor with the DX level in the bandgap. In addition, the effective-mass level is very deep $(100 \mathrm{meV})$.

Earlier work showed that the selenium donor shows metastable behavior as expected for DX. Becla et al. observed photoinduced persistent optical absorption in this system [5]. The current paper describes an electron paramagnetic resonance (EPR) study on similar material, AlSb:Se.

\section{Results and discussion}

The sample used in this study is a Czochralski-grown AlSb sample with a high concentration selenium doping $\left(2 \times 10^{17} \mathrm{~cm}^{-3}\right)$ [5]. This resulted in $n$-type AlSb with a high free-electron concentration at room temperature. The sample was placed in a commercial Bruker ER 200D X-band $(9.43 \mathrm{GHz})$ spectrometer and was cooled to low temperatures in an Oxford ESR 900 cryostat, operating at temperatures from 3.9 to $300 \mathrm{~K}$. The illumination was from a tungsten lamp.

After cooling in the dark, the sample becomes highly resistive and no EPR signal is observed. Apparently, the carriers freeze out into non-paramagnetic states, 
presumable DX. During illumination a strong signal arises around $g=1.949$. After switching off the light, this signal increases in intensity. This increase can be explained by the trapping of the free carriers on the $d^{0}$ state when the light is switched off. The free carriers can decrease the signal through a reduction the $Q$-factor and screening the interior of the sample from the resonating microwaves. The signal after illumination, as shown in Fig. 1, persists undiminished for at least several hours.

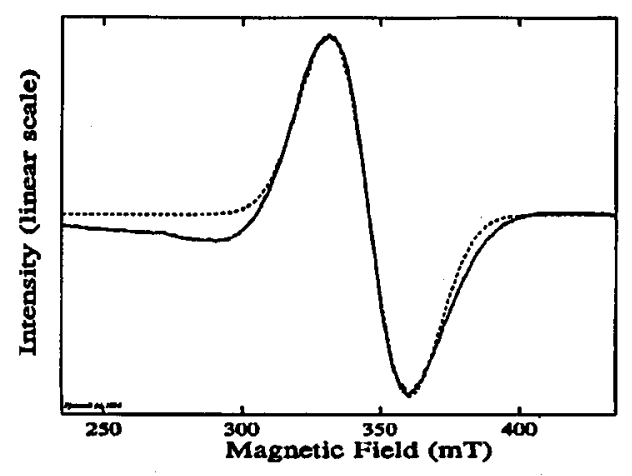

Fig. 1. Isotropic EPR spectrum of AlSb:Se after illumination. The dashed line is a Gaussian-line simulation with $g=1.949$ and a peak-to-peak width of $29 \mathrm{mT}$.

It is known that the DX center can thermally relax to the ground state at temperatures higher than ca. 100-120 K. Figure 2 shows the thermal quenching behavior of the EPR signal. To obtain an experimental point in this picture, the following procedure was followed: First the temperature was raised from $14 \mathrm{~K}$ to the indicated temperature. The sample was kept at this point for about 5 minutes, after which the temperature was restored to $14 \mathrm{~K}$, and the EPR signal intensity was measured. This procedure was used in order to avoid secondary effects, like temperature dependences of the $Q$-factor, paramagnetic relaxation times $T_{1}$ and $T_{2}$, and Boltzmann distribution over the magnetic sublevels since they can affect the signal dramatically. In this way the signal is proportional to the concentration of the paramagnetic centers. It is clear from Fig. 2 that at temperatures below $115 \mathrm{~K}$ no relaxation occurs. In the temperature $115-130 \mathrm{~K}$ the signal drops, and the original state of absence of paramagnetism is recovered.

The important conclusion is that the absence of EPR signal after cooling in the dark (and subsequent observation after illumination) is a direct proof for the negative-U model. Illumination with light transfers the systems to the hydrogenic donors, which are paramagnetic. The observed thermal quenching of the persistent EPR signal is very similar to the thermal quenching of the persistent photoconductivity in other materials (see, for example Ref. [6].

An analysis of the lineshape [7] reveals that it is Gaussian. This excludes the possibility that the line broadening is dominated by lifetime effects, which would result in a Lorentzian lineshape. Because the effective-mass state is so localized $(100 \mathrm{meV} \rightarrow 5.6 \AA)$ the wave functions do not overlap even for very high concentra- 


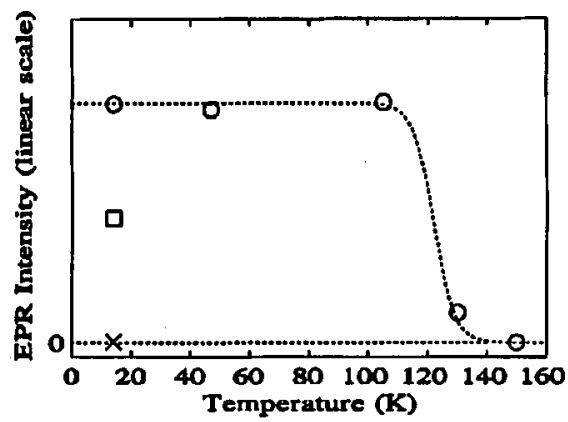

Fig. 2. Thermal quenching of the photo-induced EPR spectrum in AlSb:Se measured at $14 \mathrm{~K}$. The figure shows the EPR intensity for cooling in the dark $(x)$, during illumination (प), and after switching off the light (o). To return the sample to the nonparamagnetic state it has to be heated to a temperature above 115-130 K.

tions of the defect and no change of the lifetime (and linewidth [8]) is expected. The very large linewidth of $29 \mathrm{mT}$ peak-to-peak is not uncommon in III-V semiconductors, and is in most cases due to the fact that all stable isotopes of group-III and group- $V$ elements have nuclear spin interacting with the paramagnetic electron, and broadening of the line. In the framework of the theory of linear combination of atomic orbitals (LCAO) the hyperfine interaction is linearly proportional to the amount of the paramagnetic wave function at the site of the nucleus in question, and the element-specific interaction constant [9]. We can easily calculate the density of the wave function on the various shells of atoms. As discussed above, the paramagnetic state is an effective-mass-like state around the selenium atom substituting for an antimony cation. To find the amount of electron on each atom the square of the effective-mass wave function $\psi_{s}(r)=\left(1 / \pi a_{1}^{3 / 2}\right) \exp \left(-r / a_{1}\right)$ is evaluated at the position of the nucleus and then multiplied by the volume per atom (19.3 $\AA^{3}$ for $\left.\mathrm{AlSb}\right)$.

Inspired by this, a simulation was made with densities on the first four shells. The resulting linewidth was $73 \mathrm{mT}$. This is a factor 2.5 higher than the experimentally observed value. There may be several reasons for this deviation: (1) Not all paramagnetic electrons are in atomic s-orbitals as assumed above, but resides partly in higher orbitals (which cause less HF interaction) or in the voids of the crystal; (2) Central-core corrections can make the wave function deviate significantly from the spherical effective mass shape. This is very likely, since the effective mass electron is so localized in AlSb (viz. $a_{1}=5.6 \AA$, comparable with the lattice constant) that the host lattice does not behave as an isotropic dielectric medium, but rather has structure. In view of this, the observed linewidth is comparable to the simulated linewidth based on an effective-mass wave function.

This work was supported by the Director, Office of Energy Research, Office of Basic Energy Sciences, Materials Science Division of the U.S. Department of Energy, No. DE-AC03-76SF00098. 


\section{References}

[1] D.V. Lang, R.A. Logan, Phys. Rev. Lett. 39, 635 (1977).

[2] M.G. Craford, G.E. Stillman, N. Holonyak Jr., J.A. Rossi, Phys. Rev. 168, 867 (1968); K. Khachaturian, M. Kaminska, E.R. Weber, P. Becla, R.A. Street, Phys. Rev. B 40, 6304 (1989); J.A. Wolk, W. Walukiewicz, M.L. Thewalt, E.E. Haller, Phys. Rev. Lett. 68, 3619 (1992).

[3] D.J. Chadi, K.J. Chang, Phys. Rev. Lett. 61, 873 (1988).

[4] Y. Toyozawa, Physica B 116, 7 (1983).

[5] P. Becla, A. Witt, J. Lagowski, W. Walukiewicz, to be published.

[6] K. Khachaturian, D.D. Awschalom, J.R. Rosen, E.R. Weber, Phys. Rev. Lett. 63, 1311 (1989).

[7] R.S. Alger, Electron Paramagnetic Resonance Techniques and Application, Interscience Publ., New York 1968.

[8] H.J. von Bardeleben, J.C. Bourgoin, P. Basmaji, P. Gibart, Mat. Res. Soc. Proc. 148, 439 (1989).

[9] J.R. Morton, K.F. Preston, J. Magn. Res. 30, 577 (1978). 